\title{
ACTIVE CONTROLlER DESIGN FOR THE HYBRID SYNCHRONIZATION OF HYPERCHAOTIC XU AND HYPERCHAOTIC Li SYSTEMS
}

\author{
Sundarapandian Vaidyanathan ${ }^{1}$ \\ ${ }^{1} \mathrm{R} \& \mathrm{D}$ Centre, Vel Tech University \\ Avadi, Chennai-600 062, Tamil Nadu, INDIA \\ sundarvtuegmail.com
}

\begin{abstract}
The synchronization of chaotic systems treats a pair of chaotic systems, which are usually called as master and slave systems. In the chaos synchronization problem, the goal of the design is to synchronize the states of master and slave systems asymptotically. In the hybrid synchronization design of master and slave systems, one part of the systems, viz. their odd states, are completely synchronized (CS), while the other part, viz. their even states, are completely anti-synchronized (AS) so that CS and AS co-exist in the process of synchronization. This research work deals with the hybrid synchronization of hyperchaotic Xi systems (2009) and hyperchaotic Li systems (2005). The main results of this hybrid research work are established with Lyapunov stability theory. MATLAB simulations of the hybrid synchronization results are shown for the hyperchaotic Xu and Li systems.
\end{abstract}

\section{KEYWORDS}

Hybrid Synchronization, Active Control, Chaos, Hyperchaos, Hyperchaotic Systems.

\section{INTRODUCTION}

Hyperchaotic systems are nonlinear chaotic systems with two or more positive Lyapunov exponents. These systems have a lot of miscellaneous applications in Engineering. The hyperchaotic system was first discovered by the German scientist, O.E. Rössler ([1], 1979).

Hyperchaotic systems possess salient features like high level of security, high level of capacity and high level of efficiency. Hence, the hyperchaotic systems have important applications in areas like neural networks [2], oscillators [3], communication [4-5], encryption [6], synchronization [7], etc.

We note that many methods have been devised for the synchronization of chaotic systems. Some important methods can be cited as Ott-Grebogi-Yorke method [8], Pecora-Carroll method [9], backstepping method [10-12], sliding control method [13-15], active control method [16-18], adaptive control method [19-20], sampled-data control method [21], delayed feedback method [22], etc.

We describe the hybrid synchronization of a pair of chaotic systems called the master and slave systems as follows. Here, one part of the systems, viz. the odd states, are completely 
synchronized (CS) and the other part of the systems, viz. the even states, are anti-synchronized so that both CS and AS can exist together in the hybrid synchronization of the two systems.

This paper focuses upon active controller design for the hybrid synchronization of hyperchaotic $\mathrm{Xu}$ systems ([23], 2009) and hyperchaotic Li systems ([24], 2005). The main results derived in this paper have been proved using stability theorems of Lyapunov stability theory [25].

\section{Active Control Methodology}

The master system is described by the chaotic dynamics

$$
\dot{x}=A x+f(x)
$$

where $A$ is the $n \times n$ matrix of the system parameters and $f: R^{n} \rightarrow R^{n}$ is the nonlinear part. The slave system is described by the chaotic dynamics

$$
\dot{y}=B y+g(y)+u
$$

where $B$ is the $n \times n$ matrix of the system parameters, $g: R^{n} \rightarrow R^{n}$ is the nonlinear part and $u \in R^{n}$ is the active controller to be designed.

Let us define the hybrid synchronization error as

$$
e_{i}=\left\{\begin{array}{cc}
y_{i}-x_{i}, & \text { if } i \text { is odd } \\
y_{i}+x_{i}, & \text { if } i \text { is even }
\end{array}\right.
$$

We can calculate the error dynamics as

$$
\dot{e}_{i}= \begin{cases}\sum_{j=1}^{n}\left(b_{i j} y_{j}-a_{i j} x_{j}\right)+g_{i}(y)-f_{i}(x)+u_{i} & \text { if } i \text { is odd } \\ \sum_{j=1}^{n}\left(b_{i j} y_{j}+a_{i j} x_{j}\right)+g_{i}(y)+f_{i}(x)+u_{i} & \text { if } i \text { is even }\end{cases}
$$

As mentioned earlier, the research goal is to find a controller $u$ so that

$$
\lim _{t \rightarrow \infty}\|e(t)\|=0 \text { for all values of } e(0) \in R^{n}
$$

We apply the matrix Lyapunov method as our methodology.

Hence, we consider a candidate Lyapunov function

$$
V(e)=e^{T} P e,
$$

where $P$ is a positive definite $n \times n$ matrix.

We remark that $V: R^{n} \rightarrow R$ is a positive definite function.

If we find a feedback controller $u$ so that 


$$
\dot{V}(e)=-e^{T} Q e,
$$

where $Q$ is a positive definite matrix, then $\dot{V}: R^{n} \rightarrow R$ is a negative definite function.

Thus, the error dynamics (4) is globally exponentially stable by Lyapunov stability theory [25]. Hence, the states of the chaotic systems (1) and (2) will be globally and exponentially hybrid synchronized for all initial conditions $x(0), y(0) \in R^{n}$.

\section{HYPERCHAOTIC SYSTEMS}

The hyperchaotic Xu system ([23], 2009) has the 4-D dynamics

$$
\begin{aligned}
& \dot{x}_{1}=a\left(x_{2}-x_{1}\right)+x_{4} \\
& \dot{x}_{2}=b x_{1}+r x_{1} x_{3} \\
& \dot{x}_{3}=-c x_{3}-x_{1} x_{2} \\
& \dot{x}_{4}=x_{1} x_{3}-d x_{2}
\end{aligned}
$$

where $a, b, c, r, d$ are constant, positive parameters of the system.

The Xu system (8) exhibits a hyperchaotic attractor when the system parameters are taken as

$$
a=10, \quad b=40, c=2.5, \quad r=16, \quad d=2
$$

The Lyapunov exponents of the 4-D system (8) for the values of parameters in (9) are

$$
\lambda_{1}=1.0088, \quad \lambda_{2}=0.1063, \quad \lambda_{3}=0, \quad \lambda_{4}=-13.6191
$$

Note that there are two positive Lyapunov exponents in (10). This shows that the 4-D Xu system (8) is hyperchaotic for the parametric values (9).

The strange attractor of the hyperchaotic Xu system is displayed in Figure 1.

The hyperchaotic Li system ([24], 2005) has the 4-D dynamics

$$
\begin{aligned}
& \dot{x}_{1}=\alpha\left(x_{2}-x_{1}\right)+x_{4} \\
& \dot{x}_{2}=\delta x_{1}-x_{1} x_{3}+\gamma x_{2} \\
& \dot{x}_{3}=-\beta x_{3}+x_{1} x_{2} \\
& \dot{x}_{4}=x_{2} x_{3}+\varepsilon x_{4}
\end{aligned}
$$

where $\alpha, \beta, \gamma, \delta, \varepsilon$ are constant, positive parameters of the system.

The Li system (11) exhibits a hyperchaotic attractor when the parameter values are taken as

$$
\alpha=35, \quad \beta=3, \quad \gamma=12, \quad \delta=7, \quad \varepsilon=0.58
$$

The Lyapunov exponents of the 4-D system (11) for the values of the parameters in (12) are

$$
\lambda_{1}=0.5011, \quad \lambda_{2}=0.1858, \quad \lambda_{3}=0, \quad \lambda_{4}=-26.1010
$$

Note that there are two positive Lyapunov exponents in (13). This shows that the 4-D Li system (11) is hyperchaotic for the parametric values (12). 
The strange attractor of the hyperchaotic Li system is displayed in Figure 2.
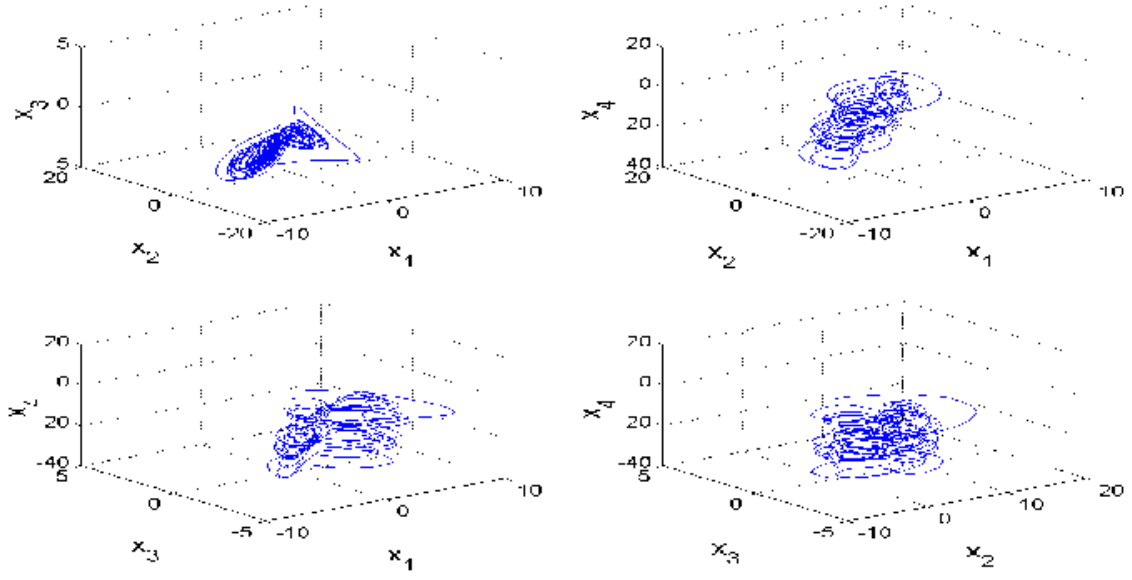

Figure 1. The State Portrait of the Hyperchaotic Xu System
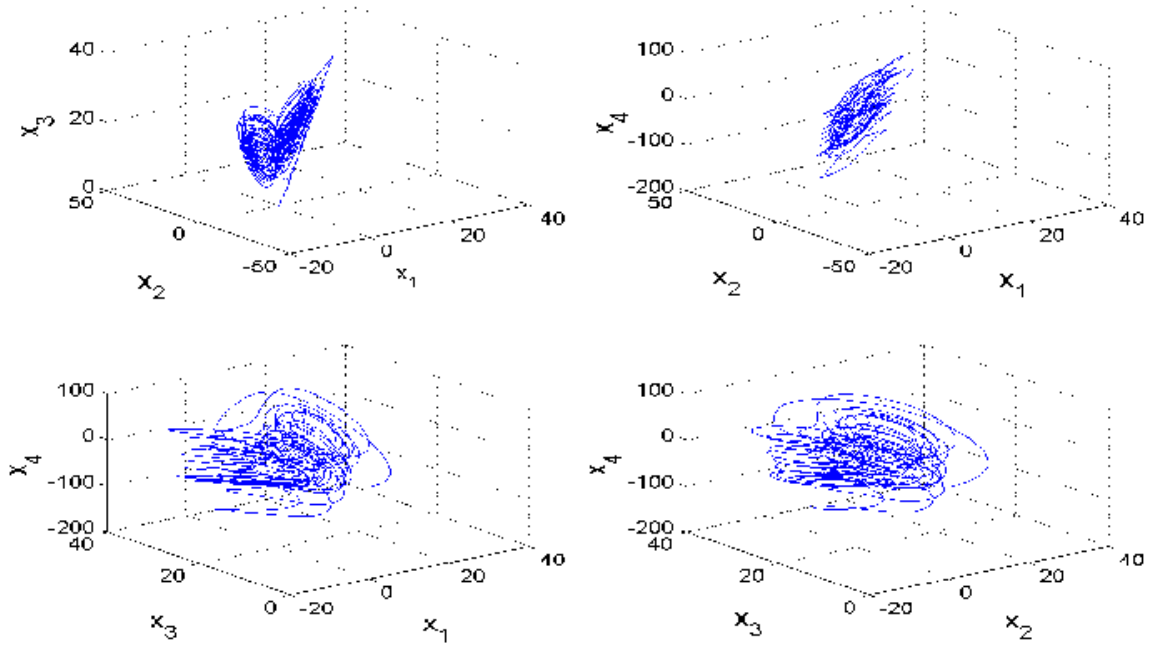

Figure 2. The State Portrait of the Hyperchaotic Li System

\section{ACtive Control Design for the Hybrid Synchronization of HYPERCHAOTIC XU SYSTEMS}

In this section, we design an active controller for the hybrid synchronization of two identical hyperchaotic Xu systems (2009) and establish our main result using Lyapunov stability theory. The hyperchaotic Xu system is taken as the master system. The dynamics of the master system is given by 


$$
\begin{aligned}
& \dot{x}_{1}=a\left(x_{2}-x_{1}\right)+x_{4} \\
& \dot{x}_{2}=b x_{1}+r x_{1} x_{3} \\
& \dot{x}_{3}=-c x_{3}-x_{1} x_{2} \\
& \dot{x}_{4}=x_{1} x_{3}-d x_{2}
\end{aligned}
$$

where $a, b, c, d$ are positive parameters of the system and $x \in R^{4}$ is the state of the system.

The hyperchaotic Xu system is also taken as the slave system. The dynamics of the slave system is given by

$$
\begin{aligned}
& \dot{y}_{1}=a\left(y_{2}-y_{1}\right)+y_{4}+u_{1} \\
& \dot{y}_{2}=b y_{1}+r y_{1} y_{3}+u_{2} \\
& \dot{y}_{3}=-c y_{3}-y_{1} y_{2}+u_{3} \\
& \dot{y}_{4}=y_{1} y_{3}-d y_{2}+u_{4}
\end{aligned}
$$

where $y \in R^{4}$ is the state and $u_{1}, u_{2}, u_{3}, u_{4}$ are the active controllers to be designed.

We consider the hybrid synchronization problem for the systems (14) and (15).

Hence, the hybrid synchronization error $e$ is defined as follows:

$$
\begin{aligned}
& e_{1}=y_{1}-x_{1} \\
& e_{2}=y_{2}+x_{2} \\
& e_{3}=y_{3}-x_{3} \\
& e_{4}=y_{4}+x_{4}
\end{aligned}
$$

When we calculate the error dynamics, we obtain

$$
\begin{aligned}
& \dot{e}_{1}=a\left(e_{2}-e_{1}\right)+e_{4}-2 a x_{2}-2 x_{4}+u_{1} \\
& \dot{e}_{2}=b e_{1}+2 b x_{1}+r\left(y_{1} y_{3}+x_{1} x_{3}\right)+u_{2} \\
& \dot{e}_{3}=-c e_{3}-y_{1} y_{2}+x_{1} x_{2}+u_{3} \\
& \dot{e}_{4}=-d e_{2}+y_{1} y_{3}+x_{1} x_{3}+u_{4}
\end{aligned}
$$

Let the active controller for achieving hybrid synchronization be taken as

$$
\begin{aligned}
& u_{1}=-a\left(e_{2}-e_{1}\right)-e_{4}+2 a x_{2}+x_{4}-k_{1} e_{1} \\
& u_{2}=-b e_{1}-2 b x_{1}-r\left(y_{1} y_{3}+x_{1} x_{3}\right)-k_{2} e_{2} \\
& u_{3}=c e_{3}+y_{1} y_{2}-x_{1} x_{2}-k_{3} e_{3} \\
& u_{4}=d e_{2}-y_{1} y_{3}-x_{1} x_{3}-k_{4} e_{4}
\end{aligned}
$$

where $k_{i},(i=1,2,3,4)$ are constants having positive values.

The error dynamics is simplified by the substitution of (18) into (17), and we obtain 


$$
\begin{aligned}
& \dot{e}_{1}=-k_{1} e_{1} \\
& \dot{e}_{2}=-k_{2} e_{2} \\
& \dot{e}_{3}=-k_{3} e_{3} \\
& \dot{e}_{4}=-k_{4} e_{4}
\end{aligned}
$$

Theorem 4.1 The active control law defined by Eq. (18) achieves global and exponential hybrid synchronization of the identical hyperchaotic Xu systems (14) and (15) for all initial conditions $x(0), y(0) \in R^{4}$.

Proof. The proof is via Lyapunov stability theory [25] for global exponential stability. As a candidate Lyapunov function, we consider the quadratic function defined by

$$
V(e)=\frac{1}{2} e^{T} e=\frac{1}{2}\left(e_{1}^{2}+e_{2}^{2}+e_{3}^{2}+e_{4}^{2}\right)
$$

Note that $V$ is a positive definite function on $R^{4}$.

Next, we differentiate (18) along the trajectories of (17).

A simple calculation gives

$$
\dot{V}(e)=-k_{1} e_{1}^{2}-k_{2} e_{2}^{2}-k_{3} e_{3}^{2}-k_{4} e_{4}^{2}
$$

Note that $\dot{V}$ is a negative definite function on $R^{4}$.

By Lyapunov stability theory [25], the error dynamics (19) is globally exponentially stable for all values of $e(0) \in R^{4}$.

This completes the proof.

Next, we illustrate our hybrid synchronization results with MATLAB simulations.

The classical fourth order Runge-Kutta method with time-step $h=10^{-8}$ has been applied to solve the hyperchaotic Xu systems (14) and (15) with the active nonlinear controller (18).

The feedback gains in the active controller (18) are chosen as follows.

We take $k_{i}=5,(i=1,2,3,4)$.

The parameters of the hyperchaotic Xu systems are taken as in the hyperchaotic case, i.e.

$$
a=10, \quad b=40, \quad c=2.5, \quad r=16, \quad d=2
$$

For simulations, the initial conditions of the hyperchaotic Xu system (14) are chosen as

$$
x_{1}(0)=21, x_{2}(0)=7, x_{3}(0)=22, x_{4}(0)=-14
$$

Also, the initial conditions of the hyperchaotic Xu system (15) are chosen as

$$
y_{1}(0)=12, \quad y_{2}(0)=-11, \quad y_{3}(0)=-20, \quad y_{4}(0)=27
$$

Figure 3 depicts the hybrid synchronization of the identical hyperchaotic Xu systems. 
International Journal of Information Technology, Modeling and Computing (IJITMC) Vol.1, No.2, May 2013

Figure 4 depicts the time-history of the anti-synchronization errors $e_{1}, e_{2}, e_{3}, e_{4}$.
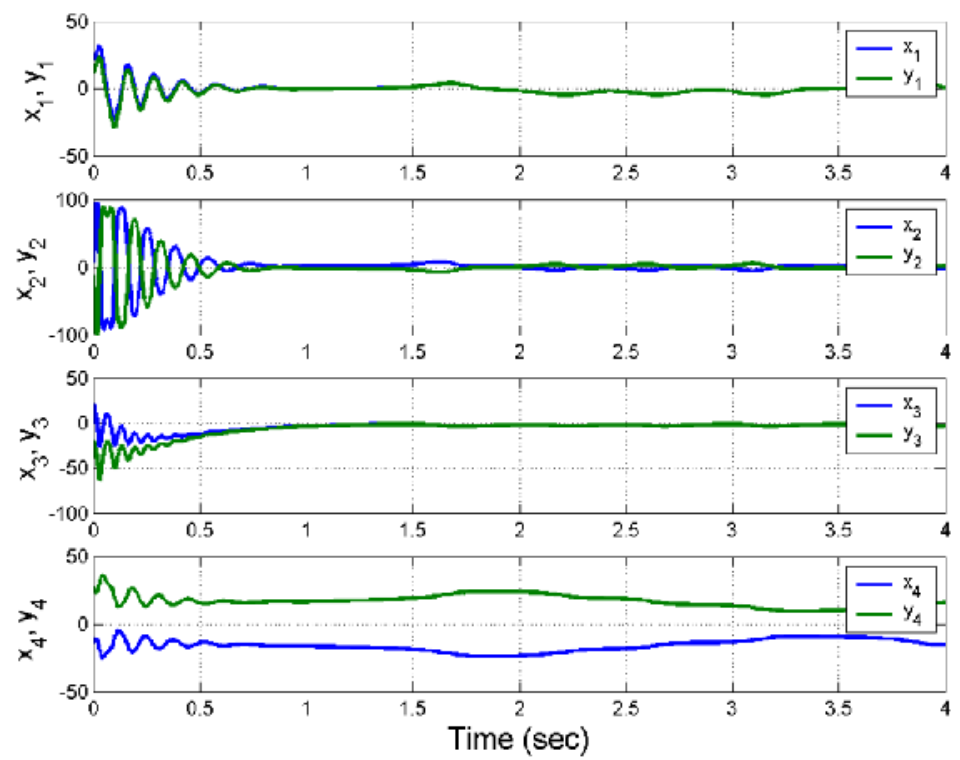

Figure 3. Hybrid Synchronization of Identical Hyperchaotic Xu Systems

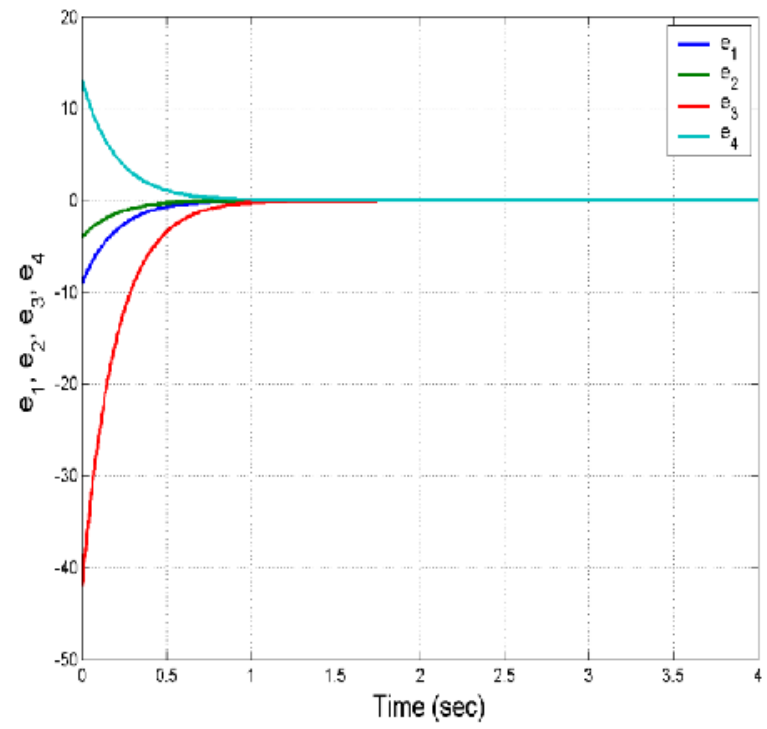

Figure 4. Time-History of the Hybrid Synchronization Errors $e_{1}, e_{2}, e_{3}, e_{4}$ 


\section{Active Controller Design for the Hybrid Synchronization DESIGN OF HYPERCHAOTIC Li SYSTEMS}

This section deals with the design of an active controller for the hybrid synchronization of two identical hyperchaotic Li systems (2005). In this section, we establish our main result using Lyapunov stability theory.

The hyperchaotic Li system is taken as the master system. Thus, the dynamics of the master system is given by

$$
\begin{aligned}
& \dot{x}_{1}=\alpha\left(x_{2}-x_{1}\right)+x_{4} \\
& \dot{x}_{2}=\delta x_{1}-x_{1} x_{3}+\gamma x_{2} \\
& \dot{x}_{3}=-\beta x_{3}+x_{1} x_{2} \\
& \dot{x}_{4}=x_{2} x_{3}+\varepsilon x_{4}
\end{aligned}
$$

where $\alpha, \beta, \gamma, \delta, \varepsilon$ are positive parameters of the system and $x \in R^{4}$ is the state of the system. The hyperchaotic Li system is taken as the slave system. The dynamics of the slave system is given by

$$
\begin{aligned}
& \dot{y}_{1}=\alpha\left(y_{2}-y_{1}\right)+y_{4}+u_{1} \\
& \dot{y}_{2}=\delta y_{1}-y_{1} y_{3}+\gamma y_{2}+u_{2} \\
& \dot{y}_{3}=-\beta y_{3}+y_{1} y_{2}+u_{3} \\
& \dot{y}_{4}=y_{2} y_{3}+\varepsilon y_{4}+u_{4}
\end{aligned}
$$

where $y \in R^{4}$ is the state and $u_{1}, u_{2}, u_{3}, u_{4}$ are the active controllers to be designed.

For the hybrid synchronization, the error $e$ is defined as

$$
\begin{aligned}
& e_{1}=y_{1}-x_{1} \\
& e_{2}=y_{2}+x_{2} \\
& e_{3}=y_{3}-x_{3} \\
& e_{4}=y_{4}+x_{4}
\end{aligned}
$$

We obtain the error dynamics as

$$
\begin{aligned}
& \dot{e}_{1}=\alpha\left(e_{2}-e_{1}\right)+e_{4}-2 \alpha x_{2}-2 x_{4}+u_{1} \\
& \dot{e}_{2}=\delta e_{1}+2 \delta x_{1}+\gamma e_{2}-y_{1} y_{3}-x_{1} x_{3}+u_{2} \\
& \dot{e}_{3}=-\beta e_{3}+y_{1} y_{2}-x_{1} x_{2}+u_{3} \\
& \dot{e}_{4}=\varepsilon e_{4}+y_{2} y_{3}+x_{2} x_{3}+u_{4}
\end{aligned}
$$

We choose the active controller for achieving hybrid synchronization as 


$$
\begin{aligned}
& u_{1}=-\alpha\left(e_{2}-e_{1}\right)-e_{4}+2 \alpha x_{2}+2 x_{4}-k_{1} e_{1} \\
& u_{2}=-\delta e_{1}-2 \delta x_{1}-\gamma e_{2}+y_{1} y_{3}+x_{1} x_{3}-k_{2} e_{2} \\
& u_{3}=\beta e_{3}-y_{1} y_{2}+x_{1} x_{2}-k_{3} e_{3} \\
& u_{4}=-\varepsilon e_{4}-y_{2} y_{3}-x_{2} x_{3}-k_{4} e_{4}
\end{aligned}
$$

where $k_{i},(i=1,2,3,4)$ are positive gains.

The error dynamics is simplified by the substitution of (26) into (25), and we obtain

$$
\begin{aligned}
& \dot{e}_{1}=-k_{1} e_{1} \\
& \dot{e}_{2}=-k_{2} e_{2} \\
& \dot{e}_{3}=-k_{3} e_{3} \\
& \dot{e}_{4}=-k_{4} e_{4}
\end{aligned}
$$

Theorem 5.1 The active control law defined by Eq. (26) achieves global and exponential hybrid synchronization of the identical hyperchaotic Li systems (22) and (23) for all initial conditions $x(0), y(0) \in R^{4}$.

Proof. The proof is via Lyapunov stability theory [25] for global exponential stability. As a candidate Lyapunov function, we take the quadratic function

$$
V(e)=\frac{1}{2} e^{T} e=\frac{1}{2}\left(e_{1}^{2}+e_{2}^{2}+e_{3}^{2}+e_{4}^{2}\right)
$$

Note that $V$ is a positive definite function on $R^{4}$.

Next, we differentiate (26) along the trajectories of (25). A simple calculation gives

$$
\dot{V}(e)=-k_{1} e_{1}^{2}-k_{2} e_{2}^{2}-k_{3} e_{3}^{2}-k_{4} e_{4}^{2}
$$

Note that $\dot{V}$ is a negative definite function on $R^{4}$.

Hence, by Lyapunov stability theory, the error dynamics (27) is globally exponentially stable for all values of $e(0) \in R^{4}$.

Next, we illustrate our hybrid synchronization results with MATLAB simulations.

The classical fourth-order Runge-Kutta method with time-step $h=10^{-8}$ has been applied to solve the hyperchaotic Li systems (22) and (23) with the active controller defined by (26).

The feedback gains in the active controller (26) are taken as follows. Let $k_{i}=5,(i=1,2,3,4)$.

We take the parameters of the hyperchaotic Li systems as

$$
\alpha=35, \quad \beta=3, \quad \gamma=12, \quad \delta=7, \quad \varepsilon=0.58
$$

For simulations, the initial conditions of the hyperchaotic Li system (22) are chosen as

$$
x_{1}(0)=15, x_{2}(0)=-26, x_{3}(0)=-14, x_{4}(0)=5
$$


Also, the initial conditions of the hyperchaotic Li system (23) are chosen as

$$
y_{1}(0)=27, \quad y_{2}(0)=18, \quad y_{3}(0)=10, \quad y_{4}(0)=-24
$$

Figure 5 depicts the hybrid synchronization of the identical hyperchaotic Li systems.

Figure 6 depicts the time-history of the hybrid synchronization errors $e_{1}, e_{2}, e_{3}, e_{4}$.
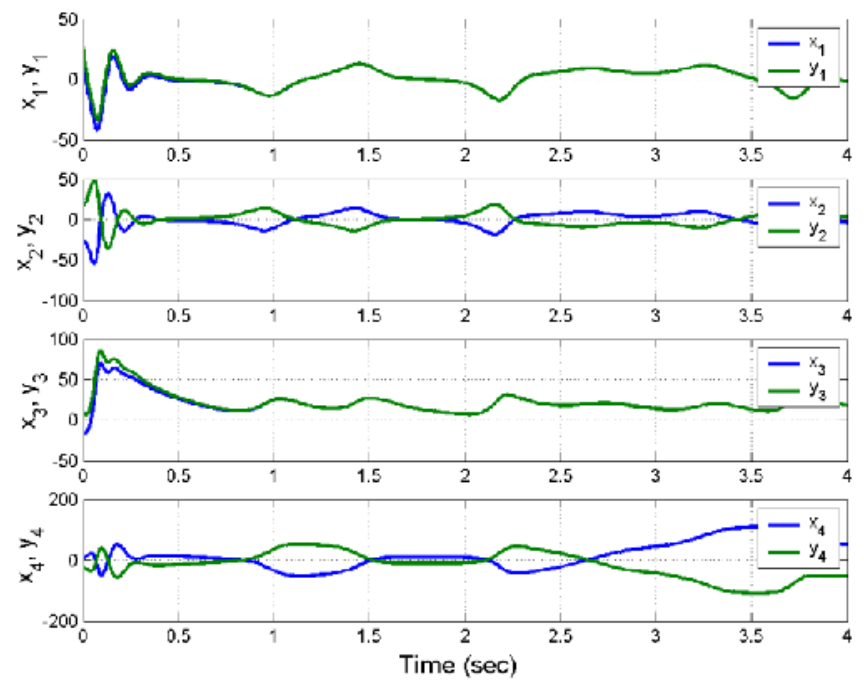

Figure 5. Hybrid Synchronization of Identical Hyperchaotic Li Systems

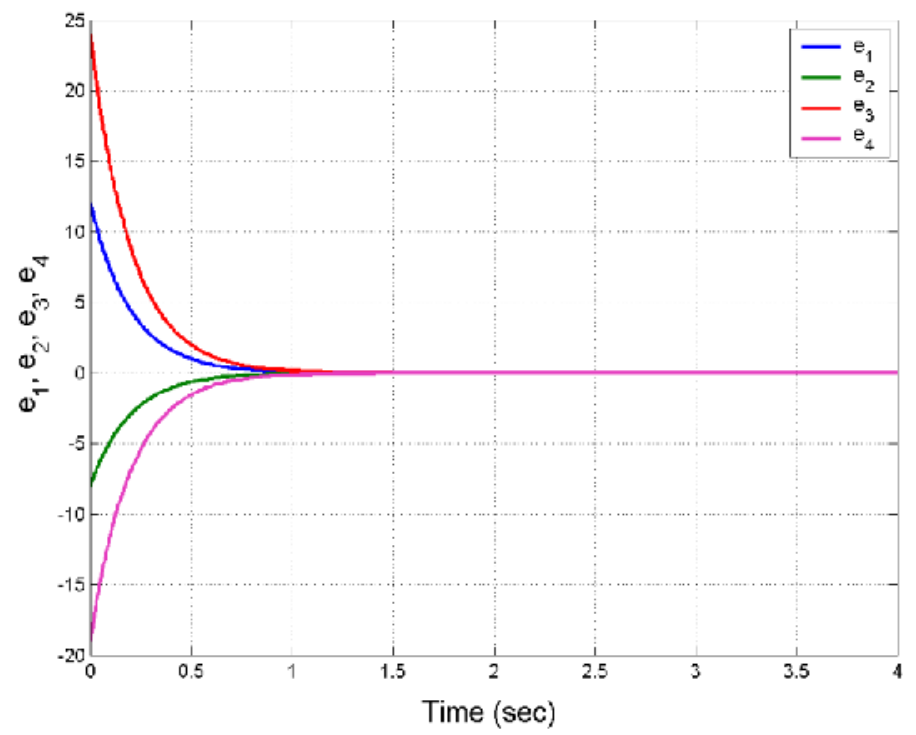

Figure 6. Time-History of the Hybrid Synchronization Errors $e_{1}, e_{2}, e_{3}, e_{4}$ 


\section{Active Controller Design for the Hybrid Synchronization of HYPERCHAOTIC XU AND HYPERCHAOTIC Li SYSTEMS}

In this section, we design an active controller for the hybrid synchronization of hyperchaotic Xu system (2009) and hyperchaotic Li system (2005) and establish our main result using Lyapunov stability theory.

The hyperchaotic $\mathrm{Xu}$ system is taken as the master system, whose dynamics is given by

$$
\begin{aligned}
& \dot{x}_{1}=a\left(x_{2}-x_{1}\right)+x_{4} \\
& \dot{x}_{2}=b x_{1}+r x_{1} x_{3} \\
& \dot{x}_{3}=-c x_{3}-x_{1} x_{2} \\
& \dot{x}_{4}=x_{1} x_{3}-d x_{2}
\end{aligned}
$$

where $a, b, c, d, r$ are positive parameters of the system and $x \in R^{4}$ is the state of the system. The hyperchaotic Li system is taken as the slave system, whose dynamics is given by

$$
\begin{aligned}
& \dot{y}_{1}=\alpha\left(y_{2}-y_{1}\right)+y_{4}+u_{1} \\
& \dot{y}_{2}=\delta y_{1}-y_{1} y_{3}+\gamma y_{2}+u_{2} \\
& \dot{y}_{3}=-\beta y_{3}+y_{1} y_{2}+u_{3} \\
& \dot{y}_{4}=y_{2} y_{3}+\varepsilon y_{4}+u_{4}
\end{aligned}
$$

where $\alpha, \beta, \gamma, \delta, \varepsilon$ are positive parameters of the system, $y \in R^{4}$ is the state and $u_{1}, u_{2}, u_{3}, u_{4}$ are the active controllers to be designed.

For the hybrid synchronization, the error $e$ is defined as

$$
\begin{aligned}
& e_{1}=y_{1}-x_{1} \\
& e_{2}=y_{2}+x_{2} \\
& e_{3}=y_{3}-x_{3} \\
& e_{4}=y_{4}+x_{4}
\end{aligned}
$$

We obtain the error dynamics as

$$
\begin{aligned}
& \dot{e}_{1}=\alpha\left(y_{2}-y_{1}\right)-a\left(x_{2}-x_{1}\right)+e_{4}-2 x_{4}+u_{1} \\
& \dot{e}_{2}=\delta y_{1}+\gamma y_{2}+b x_{1}-y_{1} y_{3}+r x_{1} x_{3}+u_{2} \\
& \dot{e}_{3}=-\beta y_{3}+c x_{3}+y_{1} y_{2}+x_{1} x_{2}+u_{3} \\
& \dot{e}_{4}=\varepsilon y_{4}-d x_{2}+y_{2} y_{3}+x_{1} x_{3}+u_{4}
\end{aligned}
$$

We choose the active controller for achieving hybrid synchronization as

$$
\begin{aligned}
& u_{1}=-\alpha\left(y_{2}-y_{1}\right)+a\left(x_{2}-x_{1}\right)-e_{4}+2 x_{4}-k_{1} e_{1} \\
& u_{2}=-\delta y_{1}-\gamma y_{2}-b x_{1}+y_{1} y_{3}-r x_{1} x_{3}-k_{2} e_{2} \\
& u_{3}=\beta y_{3}-c x_{3}-y_{1} y_{2}-x_{1} x_{2}-k_{3} e_{3} \\
& u_{4}=-\varepsilon y_{4}+d x_{2}-y_{2} y_{3}-x_{1} x_{3}-k_{4} e_{4}
\end{aligned}
$$


where $k_{i},(i=1,2,3,4)$ are positive gains.

The error dynamics is simplified by the substitution of (34) into (33), and we obtain

$$
\begin{aligned}
& \dot{e}_{1}=-k_{1} e_{1} \\
& \dot{e}_{2}=-k_{2} e_{2} \\
& \dot{e}_{3}=-k_{3} e_{3} \\
& \dot{e}_{4}=-k_{4} e_{4}
\end{aligned}
$$

Theorem 6.1 The active control law defined by Eq. (33) achieves global and exponential hybrid synchronization of the hyperchaotic Xu system (30) and hyperchaotic Li system (31) for all initial conditions $x(0), y(0) \in R^{4}$.

Proof. The proof is via Lyapunov stability theory [25] for global exponential stability. As a candidate Lyapunov function, we consider the quadratic function

$$
V(e)=\frac{1}{2} e^{T} e=\frac{1}{2}\left(e_{1}^{2}+e_{2}^{2}+e_{3}^{2}+e_{4}^{2}\right)
$$

Note that $V$ is a positive definite function on $R^{4}$.

Next, we differentiate (34) along the trajectories of (33). A simple calculation gives

$$
\dot{V}(e)=-k_{1} e_{1}^{2}-k_{2} e_{2}^{2}-k_{3} e_{3}^{2}-k_{4} e_{4}^{2}
$$

Note that $\dot{V}$ is a negative definite function on $R^{4}$.

Hence, by Lyapunov stability theory [25], the error dynamics (35) is globally exponentially stable for all values of $e(0) \in R^{4}$.

Next, we illustrate our hybrid synchronization results with MATLAB simulations.

The feedback gains in the active controller (34) are taken as follows. Let $k_{i}=5,(i=1,2,3,4)$.

The parameters of the hyperchaotic Xu and hyperchaotic Li systems are considered as in the hyperchaotic case. Thus, we take

$$
a=10, \quad b=40, \quad c=2.5, \quad r=16, \quad d=2, \alpha=35, \quad \beta=3, \quad \gamma=12, \quad \delta=7, \quad \varepsilon=0.58
$$

For simulations, the initial conditions of the hyperchaotic Xu system (30) are chosen as

$$
x_{1}(0)=27, x_{2}(0)=4, x_{3}(0)=-13, x_{4}(0)=18
$$

Also, the initial conditions of the hyperchaotic Li system (31) are chosen as

$$
y_{1}(0)=14, \quad y_{2}(0)=-28, \quad y_{3}(0)=24, \quad y_{4}(0)=-5
$$

Figure 7 depicts the hybrid synchronization of the non-identical hyperchaotic $\mathrm{Xu}$ and hyperchaotic Li systems. 
Figure 8 depicts the time-history of the hybrid synchronization errors $e_{1}, e_{2}, e_{3}, e_{4}$.
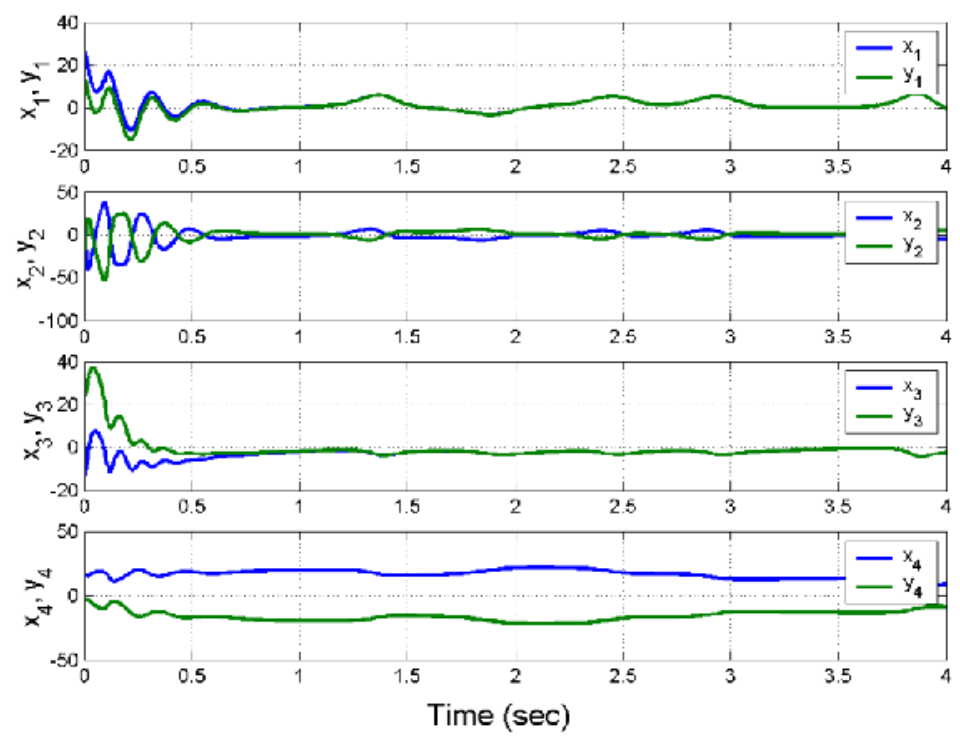

Figure 7. Hybrid Synchronization of Hyperchaotic Xu and hyperchaotic Li Systems

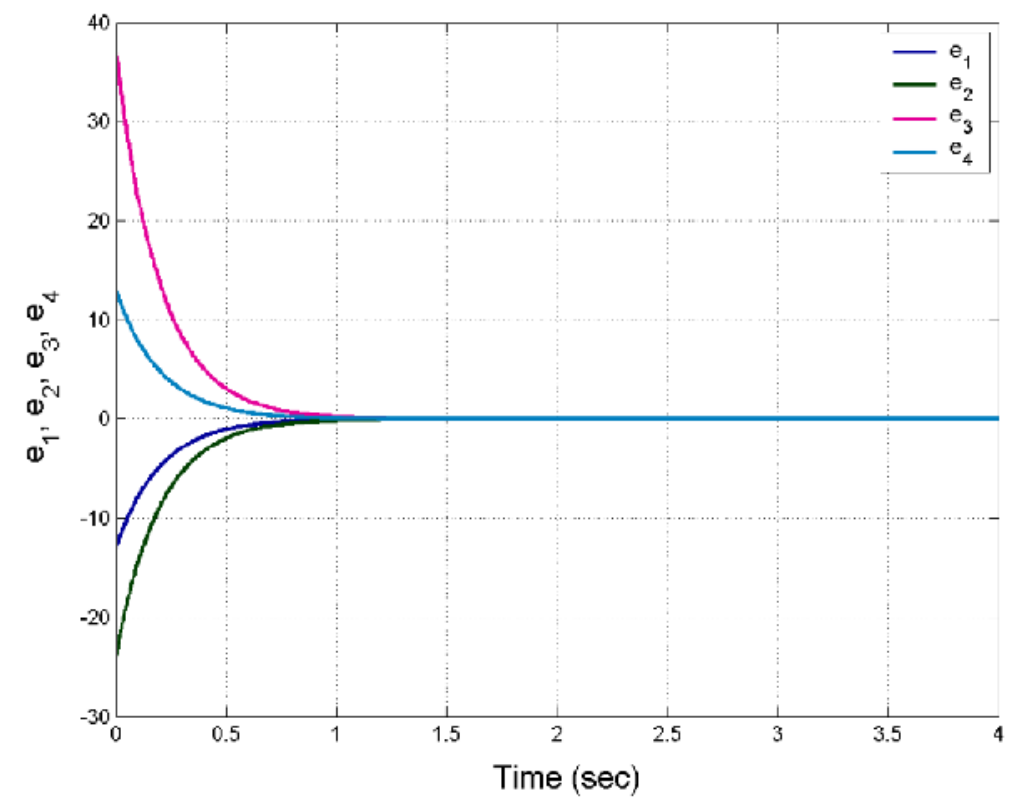

Figure 8. Time-History of the Hybrid Synchronization Errors $e_{1}, e_{2}, e_{3}, e_{4}$

\section{Conclusions}

This paper derived new results for the active controller design for the hybrid synchronization of hyperchaotic Xu systems (2009) and hyperchaotic Li systems (2005). Using Lyapunov control 
theory, active control laws were derived for globally hybrid synchronizing the states of identical hyperchaotic $\mathrm{Xu}$ systems, identical hyperchaotic Li systems and non-identical hyperchaotic Xu and $\mathrm{Li}$ systems. MATLAB simulations were displayed in detail to demonstrate the hybrid synchronization results derived in this paper for hyperchaotic $\mathrm{Xu}$ and Li systems.

\section{REFERENCES}

[1] Rössler, O.E. (1979) “An equation for hyperchaos,” Physics Letters A, Vol. 71, pp 155-157.

[2] Huang, Y. \& Yang, X.S. (2006) "Hyperchaos and bifurcation in a new class of four-dimensional Hopfield neural networks," Neurocomputing, Vol. 69, pp 13-15.

[3] Machado, L.G., Savi, M.A. \& Pacheco, P.M.C.L. (2003) "Nonlinear dynamics and chaos in coupled shape memory oscillators," International Journal of Solids and Structures, Vol. 40, No. 19, pp. 51395156.

[4] Tao, Y. (1999) "Chaotic secure communication systems - history and new results", Telecommun. Review, Vol. 9, pp 597-634.

[5] Li, C., Liao, X. \& Wong, K.W. (2005) "Lag synchronization of hyperchaos with applications to secure communications," Chaos, Solitons \& Fractals, Vol. 23, No. 1, pp 183-193.

[6] Prokhorov, M.D. \& Ponomarenko, V.I. (2008) "Encryption and decryption of information in chaotic communication systems governed by delay-differential equations," Chaos, Solitons \& Fractals, Vol. 35, No. 5, pp 871-877.

[7] Yassen, M.T. (2008) "Synchronization hyperchaos of hyperchaotic systems", Chaos, Solitons and Fractals, Vol. 37, pp 465-475.

[8] Ott, E., Grebogi, C. \& Yorke, J.A. (1990) “Controlling chaos”, Phys. Rev. Lett., Vol. 64, pp 11961199.

[9] Pecora, L.M. \& Carroll, T.L. (1990) "Synchronization in chaotic systems”, Phys. Rev. Lett., Vol. 64, pp 821-824.

[10] Bowong, S. \& Kakmeni, F.M.M. (2004) "Synchronization of uncertain chaotic systems via backstepping approach,” Chaos, Solitons \& Fractals, Vol. 21, No. 4, pp 999-1011.

[11] Suresh, R, \& Sundarapandian, V. (2012) "Global chaos synchronization of WINDMI and Coullet chaotic systems by backstepping control”, Far East J. Math. Sciences, Vol. 67, No. 2, pp 265-287.

[12] Suresh, R. \& Sundarapandian, V. (2012) "Hybrid synchronization of n-scroll Chua and Lur'e chaotic systems via backstepping control with novel feedback", Arch. Control Sciences, Vol. 22, No. 3, pp 255-278.

[13] Senejohnny, D.M. \& Delavari, H. (2012) "Active sliding observer scheme based fractional chaos synchronization," Comm. Nonlinear Sci. Numerical Simulation, Vol. 17, No. 11, pp 4373-4383.

[14] Sundarapandian, V. (2012) "Anti-synchronization of hyperchaotic Xu systems via sliding mode control”, International Journal of Embedded Systems, Vol. 2, No. 2, pp 51-61.

[15] Sundarapandian, V. (2013) "Anti-synchronizing sliding controller design for identical Pan systems," International Journal of Computational Science and Information Technology, Vol. 1, No. 1, pp 1-9.

[16] Huang, L. Feng, R. \& Wang, M. (2004) "Synchronization of chaotic systems via nonlinear control," Physics Letters A, Vol. 320, No. 4, pp 271-275.

[17] Lei, Y., Xu, W. \& Zheng, H. (2005) "Synchronization of two chaotic nonlinear gyros using active control," Physics Letters A, Vol. 343, pp 153-158.

[18] Sarasu, P. \& Sundarapandian, V. (2011) "Active controller design for generalized projective synchronization of four-scroll chaotic systems", International Journal of System Signal Control and Engineering Application, Vol. 4, No. 2, pp 26-33.

[19] Sundarapandian, V. (2012) "Adaptive control and synchronization of a generalized Lotka-Volterra system,” Vol. 1, No. 1, pp 1-12.

[20]Sundarapandian, V. (2013) "Adaptive controller and synchronizer design for hyperchaotic Zhou system with unknown parameters," Vol. 1, No. 1, pp 18-32.

[21] Zhao, J. \& Lü, J. (2006) "Using sampled-data feedback control and linear feedback synchronization in a new hyperchaotic system," Chaos, Solitons \& Fractals, Vol. 35, pp. 376-382.

[22] Ma, H., Deshmukh, V., Butcher, E. \& Averina, V. (2005) "Delayed state feedback and chaos control for time-periodic systems via a symbolic approach", Communications in Nonlinear Science and Numerical Simulation, Vol. 10, No. 5, pp 479-497. 
[23] Xu, J., Cai, G. \& Zheng, S. (2009) “A novel hyperchaotic system and its control”, Journal of Uncertain Systems, Vol. 3, No. 2, pp 137-144.

[24] Li, Y., Tang, W.K.S. \& Chen, G. (2005) "Generating hyperchaos via state feedback control", International Journal of Bifurcation and Chaos, Vol. 15, No. 10, pp 3367-3375.

[25] Hahn, W. (1967) The Stability of Motion, Springer, Berlin.

\section{AUTHOR}

Dr. V. Sundarapandian earned his D.Sc. in Electrical and Systems Engineering from Washington University, St. Louis, USA in May 1996. He is Professor and Dean of the R \& D Centre at Vel Tech Dr. RR \& Dr. SR Technical University, Chennai, Tamil Nadu, India. So far, he has published over 310 research works in refereed international journals. He has also published over 200 research papers in National and International Conferences. He has delivered Key Note Addresses at many International Conferences with IEEE and Springer Proceedings. He is an India Chair of AIRCC. He is the Editor-in-Chief of the

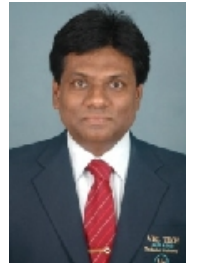
AIRCC Control Journals - International Journal of Instrumentation and Control Systems, International Journal of Control Theory and Computer Modelling, International Journal of Information Technology, Control and Automation, International Journal of Chaos, Computing, Modelling and Simulation \& International Journal of Information Technology, Modeling and Computing. His research interests are Control Systems, Chaos Theory, Soft Computing, Operations Research, Mathematical Modelling and Scientific Computing. He has published four text-books and conducted many workshops on Scientific Computing, MATLAB and SCILAB. 